\title{
Antitumor effect of extracts from moutan cortex on DLD-1 human colon cancer cells in vitro
}

\author{
GUOQING XING, ZHENYA ZHANG, JIQIANG LIU, HONGHAI HU and NORIO SUGIURA \\ Graduate School of Life and Environmental Sciences, University of Tsukuba, Tsukuba 305-8572, Japan
}

Received September 23, 2009; Accepted October 27, 2009

DOI: $10.3892 / \mathrm{mmr} \_00000218$

\begin{abstract}
Moutan cortex, the root bark of Paeonia suffruticosa Andrews (Paeoniaceae), is a traditional Chinese medicine widely used for the treatment of various diseases. In the present study, we examined the antiproliferative effect and apoptosis-inducing activity of paeonol and the crude total glycosides (CTG) extracted from moutan cortex against DLD-1 human colon cancer cells in vitro. Cell viability was measured using the WST- 8 assay. Apoptosis was detected by Hoechst 33258 fluorescence staining and flow cytometry. Paeonol and the CTG significantly reduced cell viability in a dose- and time-dependent manner. The induction of apoptosis in DLD-1 cells was characterized by morphological changes and an increased percentage of hypodiploid cells. After treatment for $48 \mathrm{~h}$ with paeonol $(400 \mu \mathrm{g} / \mathrm{ml})$ or CTG $(200 \mu \mathrm{g} / \mathrm{ml})$, the ratio of apoptotic cells reached 34.79 and $48.12 \%$, respectively. The findings obtained indicate that paeonol and the CTG extracted from moutan cortex have a significant growth-inhibitory effect on human colon cancer cells. This effect may be related to the induction of apoptosis.
\end{abstract}

\section{Introduction}

Colon cancer is amont the leading causes of premature mortality in developed countries (1-3). Recently, colon cancer rates have increased in Asia due to a rapid escalation in the adoption of westernized dietary patterns (4). Natural products have been seen as potential sources of novel anticancer drugs for the past several decades (5). Many plants have been examined to identify new and effective anti-cancer compounds, as well as to elucidate the mechanisms of cancer prevention via apoptosis.

Moutan cortex, the root bark of Paeonia suffruticosa Andrews (Paeoniaceae), has been used extensively in eastern Asian countries as a traditional Chinese medicine

Correspondence to: Dr GQ. Xing, Graduate School of Life and Environmental Sciences, University of Tsukuba, Tsukuba 305-8572, Japan

E-mail: xingguoqing@hotmail.com

Key words: moutan cortex, paeonol, glycosides, DLD-1 cell line, antiproliferative effect, apoptosis for the treatment of various diseases. Most pharmacological investigations of moutan cortex have addressed its central nervous system activities, and its anti-oxidative and sedative actions (6-10).

Paeonol (2-hydroxy-4-methoxyacetophenone) (Fig. 1), a major active component isolated from moutan cortex (11), shows extensive pharmacological activities including antioxidation, anti-inflammation and immunoregulation (12). There is a wealth of information pertaining to paeonol-induced apoptosis in various tumor cell lines (13-15). The crude total glycosides (CTG) of moutan cortex exhibit anti-inflammatory activity, immunomodulatory action, and a protective effect against liver injury (16-18). Cells undergoing apoptosis are recognized and engulfed by macrophages without damage to neighboring cells (19).

The apoptosis-inducing activities of these extracts are expected to provide a novel means of chemoprevention and chemotherapy in the treatment of cancer. However, little information is available regarding the ability of paeonol or CTG to prevent colon cancer. In the present study, we obtained clear evidence of the apoptosis-inducing and anti-proliferative effects of paeonol and CTG on DLD-1 human colon cancer cells.

\section{Materials and methods}

Materials. The human colon cancer cell line DLD-1 was obtained from the Cell Resource Center for Biomedical Research, Aging and Cancer, Tohoku University. Moutan cortex was purchased from Luoyang Huayi Biotechnology Co. (Henan, P.R. China). RPMI-1640 medium was purchased from Sigma Co. (St. Louis, MO, USA). Fetal bovine serum was purchased from CSL Limited (Parkville, Australia). A Cell Counting kit-8 was purchased from Dojin East (Tokyo, Japan). Propidium iodide (PI) was purchased from Sigma-Aldrich Co. (St. Louis, MO, USA). Hoechst 33258 was purchased from Calbiochem-Novabiochem Co. (San Diego, CA, USA).

Preparation of paeonol and CTG. Dried moutan cortex materials were ground in a mill. The resulting powder $(50 \mathrm{~g})$ was dissolved in $500 \mathrm{ml}$ of distilled water, and paeonol was extracted by steam distillation. The distillate was collected and left to cool at $4^{\circ} \mathrm{C}$ overnight, and the acicular crystal was dried in a silica gel desiccator. The purity of the paeonol used in this study was $>97 \%$, as determined by HPLC. 


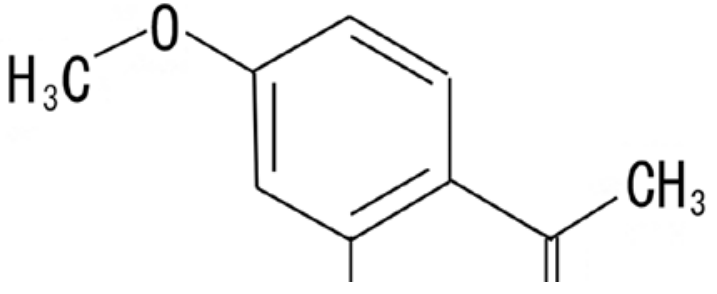

Figure 1. Chemical structure of paeonol.

The residual fraction of the paeonol extraction was combined with $400 \mathrm{ml}$ of $99 \%$ ethanol (80\% final concentration) and extracted over $2 \mathrm{~h}$ by refluxing extraction. The extract was filtered through Whatman no. 4 filter paper, then the filtrated $(400 \mathrm{ml})$ was then evaporated and lyophilized. The paeonol and CTG were dissolved in DMSO and stored at $-20^{\circ} \mathrm{C}$.

Cell proliferation assay. DLD-1 cancer cells were cultured in RPMI-1640 medium supplemented with $10 \%$ heat-inactivated fetal bovine serum and antibiotics $(100 \mathrm{U} / \mathrm{ml}$ penicillin and $100 \mu \mathrm{g} / \mathrm{ml}$ streptomycin) at $37^{\circ} \mathrm{C}$ in $5 \%(\mathrm{v} / \mathrm{v}) \mathrm{CO}_{2}$. To evaluate the effect of the extracts on the proliferation and viability of DLD-1 cancer cells, the cells were seeded at a density of $5 \times 10^{3}$ cells/well containing $100 \mu \mathrm{l}$ of culture medium in 96-well plates. After cultivation for $24 \mathrm{~h}$, the medium was replaced with fresh medium supplemented with various concentrations of the extracts and further cultivated for the indicated periods (Fig. 2). The extracts dissolved in DMSO were added to the culture medium at a final concentration of $12.5-300 \mu \mathrm{g} / \mathrm{ml}$. The final concentration of DMSO in the culture medium was $0.5 \%(\mathrm{v} / \mathrm{v})$. The control culture received only DMSO at a concentration of $0.5 \%(\mathrm{v} / \mathrm{v})$ (vehicle alone). After further incubation, $10 \mu \mathrm{l}$ of WST- 8 reagent was added to each well of a 96-well plate, followed by incubation for $3 \mathrm{~h}$ at $37^{\circ} \mathrm{C}$. The number of viable cells was determined with absorbance at $450 \mathrm{~nm}$. The wells without the extracts and the free cells (culture medium alone) were used as blanks. Data were expressed as percentages of the controls.

Morphological study of DLD-1 apoptotic cells. Cells were seeded in 6-well plates at a density of $2 \times 10^{5}$ cells $/ \mathrm{ml}$ containing $2 \mathrm{ml}$ of culture medium and cultured for $24 \mathrm{~h}$. The medium was then exchanged for one containing 100, 200 or $400 \mu \mathrm{g} / \mathrm{ml}$ of the extracts. After $48 \mathrm{~h}$ of incubation, the cells were stained with Hoechst $33258(10 \mu \mathrm{g} / \mathrm{ml})$, and changes in the nuclei were observed through a UV filter with a Leica fluorescence microscope.

Quantitative analysis of DLD-1 apoptotic cells. PI staining was used for the quantitative analysis of apoptosis. Briefly, cells were seeded in 6-well plates at a density of $2 \times 10^{5}$ cells/ $\mathrm{ml}$ and treated with the extracts as described above for the morphological study. After exposure to the extracts for the indicated periods, a total of $1-5 \times 10^{6}$ cells were harvested by trypsin treatment, washed with $\mathrm{PBS}$, and then fixed with cold $70 \%$ ethanol in PBS at $4^{\circ} \mathrm{C}$ for at least $4 \mathrm{~h}$. The cells was re-suspended in $1.0 \mathrm{ml}$ PBS for $5 \mathrm{~min}$, filtered with a 400 mesh sieve and stained with $50 \mu \mathrm{l}$ PI solution $(1.0 \mathrm{mg} / \mathrm{ml}$ in PBS) at $4^{\circ} \mathrm{C}$ for $30 \mathrm{~min}$ in the dark. DNA histograms were generated by flow cytometry (BD-LSR, BD Biosciences). Data from 10,000 cells per sample were collected, and the percentage of apoptotic cells was obtained using CellQuest software (Becton Dickinson).
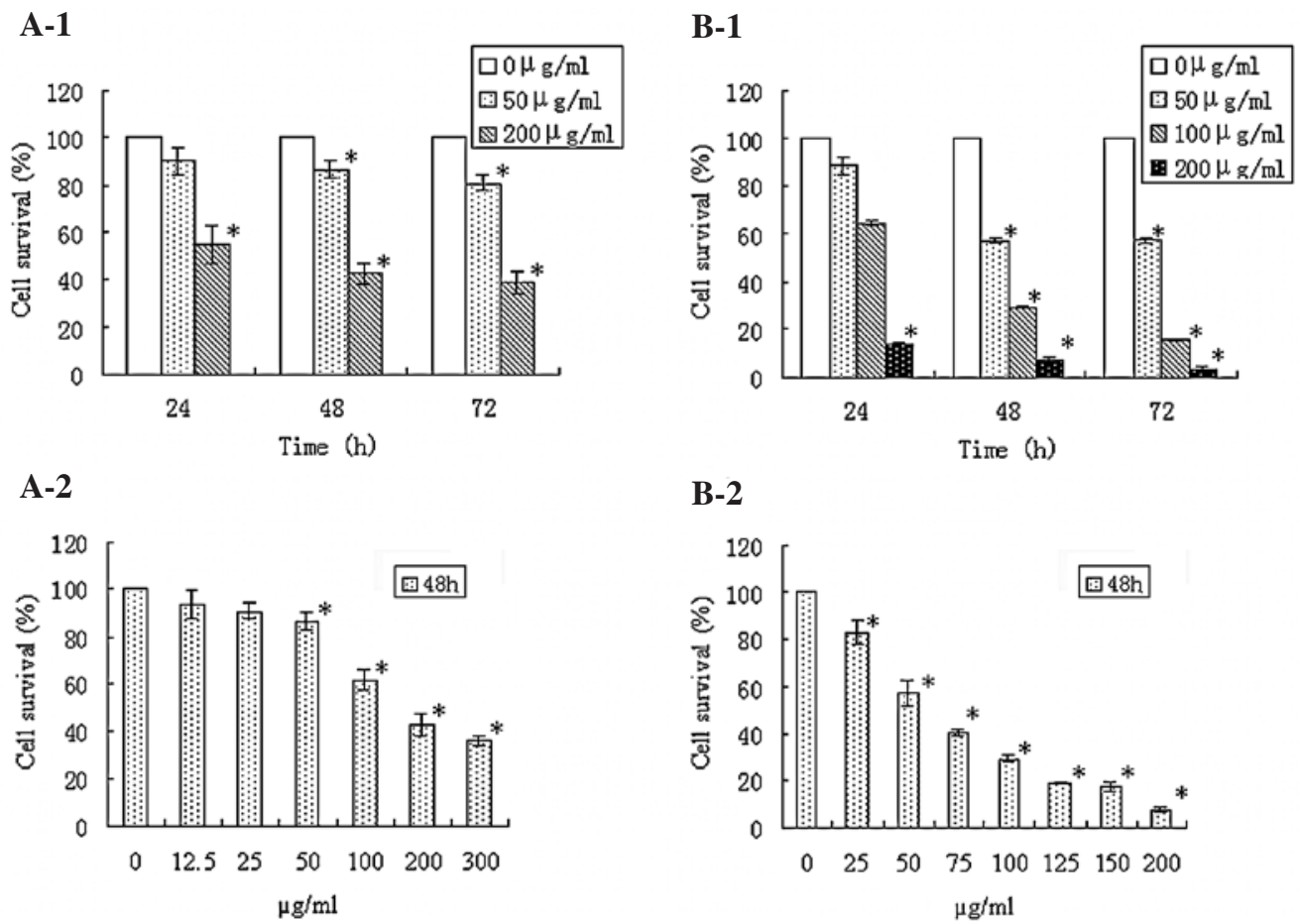

Figure 2. Paeonol and CTG inhibit the proliferation of DLD-1 cancer cells. DLD-1 cells in 96-well plates were treated with paeonol (A-1 and A-2) or CTG (B-1 and B-2) at the indicated concentrations. Cell proliferation was measured at the indicated times using the WST-8 assay. The number of viable cells was proportional to the absorbance. Data are the mean $\pm \mathrm{SD}$ of three independent experiments. *Significantly different from control values; $\mathrm{P}<0.05$. 

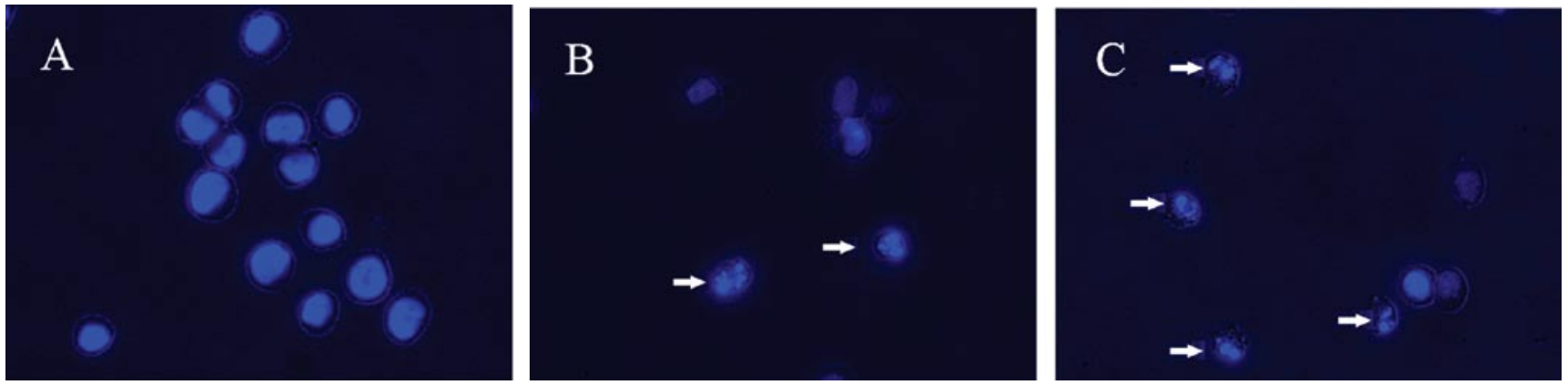

Figure 3. Apoptosis induced in DLD-1 cancer cells by paeonol or CTG. Morphological changes observed by fluorescence microscopy with Hoechst 33258 staining. DLD-1 cells were incubated for $48 \mathrm{~h}$ in the presence of (A) DMSO alone and in the presence of (B) paeonol (400 $\mu \mathrm{g} / \mathrm{ml})$ or (C) CTG (200 $\mu \mathrm{g} / \mathrm{ml})$. Arrows, apoptotic cells. Magnification x400.
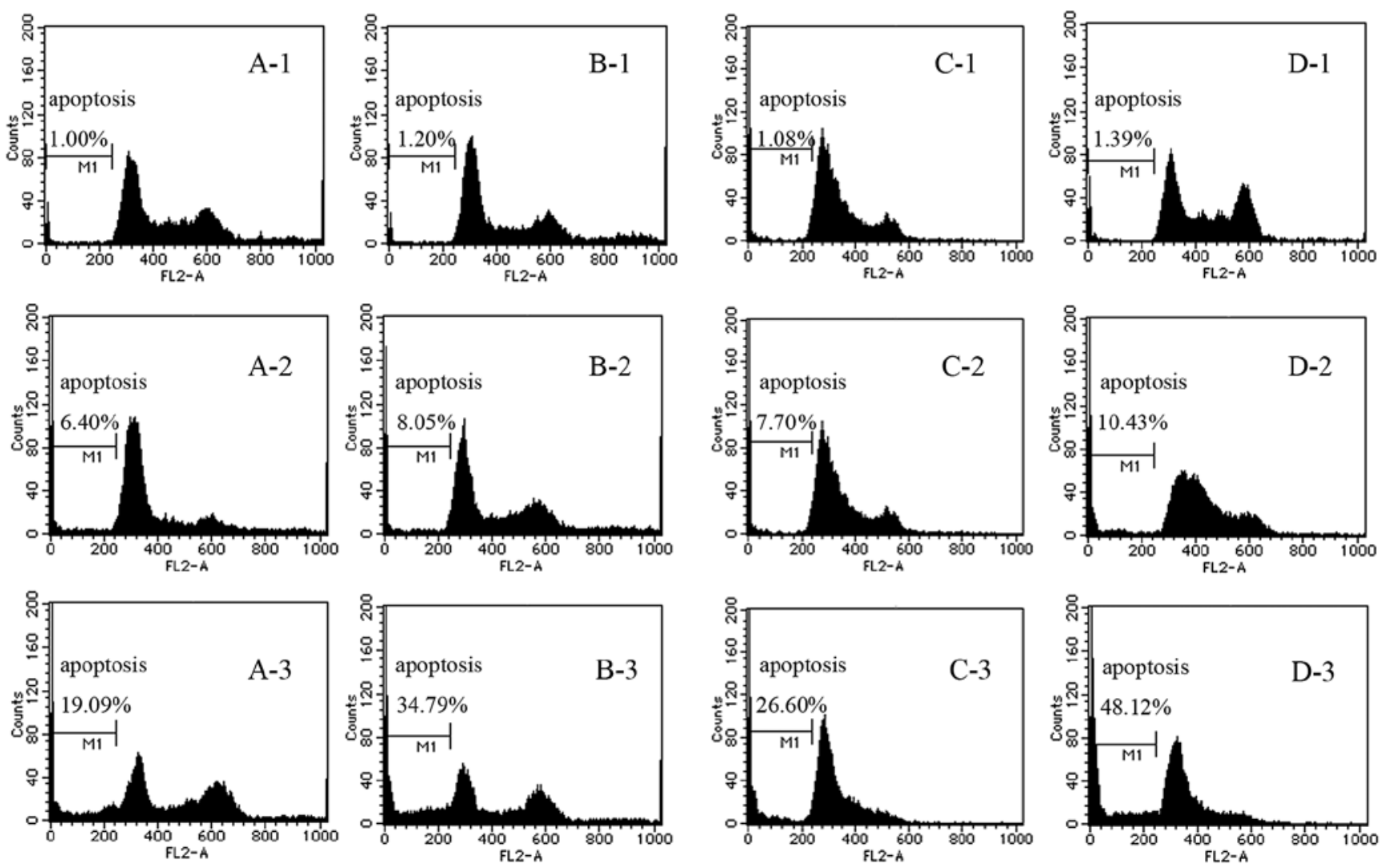

Figure 4. Percentage of apoptotic cells in the DLD-1 cell line treated with paeonol or the CTG. The percentage of apoptotic cells was obtained by flow cytometry. DLD-1 cells were incubated with 5\% DMSO (A-1, B-1, C-1 and D-1) or with $200 \mu \mathrm{g} / \mathrm{ml}$ paeonol (A-2 and B-2), $400 \mu \mathrm{g} / \mathrm{ml}$ paeonol (A-3 and B-3), $100 \mu \mathrm{g} / \mathrm{ml}$ CTG (C-2 and D-2), or $200 \mu \mathrm{g} / \mathrm{ml} \mathrm{CTG} \mathrm{(C-3} \mathrm{and} \mathrm{D-3).} \mathrm{A} \mathrm{and} \mathrm{C,} \mathrm{treatment} \mathrm{duration} \mathrm{of} 24 \mathrm{~h}$; B and D, treatment duration of $48 \mathrm{~h}$.

Statistical analysis. Values are expressed as the mean $\pm \mathrm{SD}$. The significance of differences as compared to the respective controls for each experimental test condition was assayed using the Student's t-test, with $\mathrm{P}<0.05$ or 0.01 considered significant.

\section{Results}

Antiproliferative effects. To test the effect of the extracts on the proliferation of DLD-1 cancer cells, the cells were treated with various concentrations of paeonol or CTG for different exposure times. The growth inhibition of DLD-1 cells was determined using the WST- 8 assay. The amount of yellow formazan dye generated by dehydrogenase activity in cells is directly proportional to the number of living cells in culture medium. The results indicated that the extracts isolated from moutan cortex exhibited remarkable antiproliferative effects on DLD-1 cancer cells. The viability of DLD-1 cells treated with paeonol or CTG decreased in a dose- and time-dependent manner from 24 to $72 \mathrm{~h}$ of incubation (Fig. 2). After incubation for $48 \mathrm{~h}$, the $\mathrm{IC}_{50}$ of paeonol on DLD-1 cells was $170 \mu \mathrm{g}$ / $\mathrm{ml}$, while that of the CTG was $59 \mu \mathrm{g} / \mathrm{ml}$. After $72 \mathrm{~h}$ of incubation with $200 \mu \mathrm{g} / \mathrm{ml}$ paeonol or CTG, the number of viable cells decreased to 38.78 or $3.30 \%$, respectively, compared to the control. 
Apoptotic morphological changes. To examine whether paeonol or CTG inhibits the proliferation of DLD-1 cells by inducing apoptosis, Hoechst 33258 staining was used to observe the morphological changes induced by the extracts in the cells. Control cells had round nuclei with well-distributed chromatin, whereas typical apoptotic morphology, characterized by condensed chromatin, nuclear fragmentation and the appearance of apoptotic bodies, was apparent after $48 \mathrm{~h}$ of exposure to paeonol or CTG (Fig. 3).

Quantitative analysis of apoptotic cells. To further quantitatively characterize the apoptosis process, the ratio of apoptotic cells was evaluated by fluorescence-activated cell sorting (FACS). The ratio of apoptotic cells increased significantly in a dose- and time-dependent manner compared to the control group (Fig. 4). After incubation for $48 \mathrm{~h}$ with $400 \mu \mathrm{g} / \mathrm{ml}$ paeonol or $200 \mu \mathrm{g} / \mathrm{ml} \mathrm{CTG}$, the ratio of apoptotic cells reached 34.79 or $48.12 \%$, respectively, compared to the control.

\section{Discussion}

In the current study, we used a cell viability assay, morphological observation and FACS analysis to demonstrate for the first time that paeonol and CTG remarkably reduce viability and induce apoptosis in human colon cancer DLD-1 cells.

As seen in the results, the growth of DLD-1 cells was significantly inhibited in a dose- and time-dependent manner after treatment with paeonol or CTG. A similar induction of apoptosis in human hepatoma cell lines and esophageal cancer cell lines has been reported for paeonol. Although the mechanism of the antiproliferative effect of paeonol on tumor cells is not entirely clear, it has been proposed that the induction of apoptosis is responsible for its growth inhibitory effect in vitro $(14,15)$.

Apoptosis is a physiological process controlling cell number and proliferation that helps to maintain the homeostasis of multicellular organisms (20). The induction of apoptosis is considered an attractive strategy for cancer therapy $(21,22)$. The hypothesis that failure to undergo apoptosis contributes to the development of resistance to anticancer agents has been the subject of extensive research $(22,23)$. It is believed that agents that facilitate apoptosis should improve therapeutic efficacy. In the present study, the DLD-1 cells treated with paeonol or CTG showed morphological changes typical of apoptosis and sub-G1 population accumulation. The ratio of apoptotic cells determined by FACS quantitative analysis increased in a dose- and time-dependent manner.

These effects were correspondingly reflected in the reduction of cell viability. Internucleosomal degradation of DNA due to the activation of endogenous endonuclease occurs during apoptosis. These results indicate for the first time that paeonol or CTG reduces the viability of human colon cancer cells by inducing apoptosis.

To identify the mechanism of paeonol-induced apoptosis, $\mathrm{Xu}$ et al (14) examined the expression of the Bcl-2 protein family, which is an important regulator of apoptosis (24). It was suggested that the up- or down-regulation of the Bcl-2 protein family by paeonol might be responsible for apoptosis.
CTG extracted from moutan cortex with ethanol exhibit anti-inflammatory activity, immunomodulatory action and a protective effect against liver injury (16-18). Matsuda et al examined the radical scavenging effects of seven paeonol glycosides and four monoterpene glucosides from the methanol-eluted fraction of Chinese moutan cortex, and confirmed that the galloyl group was essential for the radical scavenging effect (10). Using the Folin-Ciocalteu colorimetric method, we estimated that there are numerous polyphenols present in CTG. This is the first time the antitumor effects of CTG have been evaluated in vitro. The polyphenol and glycoside contents of CTG may contribute to its strong antitumor effect. Further research is necessary to isolate and quantify the compounds of CTG, and to investigate its antitumor mechanisms.

In conclusion, both paeonol and CTG isolated from moutan cortex decreased cell viability and induced apoptosis in the human colon cancer DLD-1 cell line, with CTG exhibiting stronger effects. Our findings indicate that paeonol and CTG potentially have chemopreventive or chemotherapeutic effects on colon cancer. The mechanisms behind the apoptosis induced by these extracts remains to be clarified.

\section{Acknowledgements}

The authors thank the Institute of Development, Aging and Cancer of Tohoku University for supplying the DLD-1 cells, and are very grateful for the use of FACS offered by the Graduate School of Comprehensive Human Sciences, University of Tsukuba.

\section{References}

1. Potter JD: Colorectal cancer: molecules and populations. J Natl Cancer Inst 91: 916-932, 1999.

2. Linsalata $M$ and Russo F: Nutritional factors and polyamine metabolism in colorectal cancer. Nutrition 24: 302-389, 2008.

3. Volate SR, Davenport DM, Muga J and Wargovich MJ: Modulation of aberrant crypt foci and apoptosis by dietary herbal supplements (quercetin, curcumin, silymarin, ginseng and rutin). Carcinogenesis 26: 1450-1456, 2005.

4. Park HJ, Kim MJ, Ha E and Chung JH: Apoptotic effect of hesperidin through caspase 3 activation in human colon cancer cells, SUN-C4. Phytomedicine 15: 147-151, 2008.

5. Hwang JH, Kim JY, Cha MR and Park HR: Effect of methanolic extract from silkworm droppings on proliferation and caspase activity in HT-29 human colon cancer cells. J Med Food 10: 467-472, 2007.

6. Ma Y, Liu J and Sun W: Effect of Moutan cortex on dynamics of blood stream in ischemic dog model. Shanxi Medicine1 3: 212-214, 1984.

7. Li Y, Zhang W, Huang L and Shi J: Pharmacological of cortex moutan and core. Chin J Chin Materia Med 22: 214-216, 1997.

8. Rho S, Chung HS, Kang M, et al: Inhibition of production of reactive oxygen species and gene expression profile by treatment of ethanol extract of moutan cortex radicis in oxidative stressed PC12 cells. Biol Pharm Bull 28: 661-666, 2005.

9. Yoshikawa M, Uchida E, Kawaguchi A, Kitagawa I and Yamahara J: Galloyl-oxypaeoniflorin, suffruticosides A, B, C and $\mathrm{D}$, five new antioxidative glycosides and suffruticoside $\mathrm{E}$, A paeonol glycoside, from Chinese moutan cortex. Chem Pharm Bull 40: 2248-2250, 1992.

10. Matsuda H, Ohta T, Kawaguchi A and Yoshikawa M: Bioactive constituents of chinese natural medicines. VI. Moutan cortex. (2): structures and radical scavenging effects of suffruticosides A, B, $\mathrm{C}, \mathrm{D}$ and $\mathrm{E}$ and galloyl-oxypaeoniflorin. Chem Pharm Bull 49: 69-72, 2001. 
11. Riley CM and Ren TC: Simple method for the determination of paeonol in human and rabbit plasma by high performance liquid chromatography using solid-phase extraction and ultraviolet detection. J Chromatogr 489: 427-432, 1989.

12. Sun YC, Shen YX and Sun GP: Advances in the studies of major pharmacological activity of paeonol. Chin Tradit Patent Med 26 : 579-582, 2004.

13. Xu SP, Sun GP, Shen YX, Wei W, Peng WR and Wang H: Antiproliferation and apoptosis induction of paeonol in HepG2 cells. World J Gastroenterol 13: 250-256, 2007.

14. Xu SP, Sun GP, Shen YX, Peng WR, Wang H and Wei W: Synergistic effect of combining paeonol and cisplatin on apoptotic induction of human hepatoma cell lines. Acta Pharmacol Sin 28: 869-878, 2008

15. Wan XA, Sun GP, Wang H, XU SP, Wang ZG and Liu SH: Synergistic effect of paeonol and cisplatin on oesophageal cancer cell lines. Dig and Liver Dis 40: 531-539, 2008.

16. Tang WL, Li J and Xu SY: Study on anti-inflammatory action of TGM. Acta Universitis Med Anhui 34: 11-13, 1999.

17. Mei Q, Wei W, Xu JM, et al: Effects of total glucosides of mudan cortex on chemical liver injury in mice. Acta Universitis Med Anhui 34: 86-88, 1999.
18. Mei Q, Wei W, Xu JM, et al: Protective effect mechanisms of total glucosides of mudan cortex on chemical liver jnjury. Chin Pharmacol Bull 15: 176-178, 1999.

19. Compto MM: A biochemical hallmark of apoptosis: Internucleosomal degradation of the genome. Cancer Metastasis Rev 11: 105-119, 1992

20. Trump BF, Berezesky IK, Chang SH and Phelps PC: The pathways of cell death: oncosis, apoptosis and necrosis. Toxicol Pathol 25: 82-88, 1997.

21. Thompson CB: Apoptosis in the pathogenesis and treatment of disease. Science 267: 1456-1462, 1995.

22. Smets LA: Programmed cell death (apoptosis) and response to anti-cancer drugs. Anticancer Drugs 5: 3-9, 1994.

23. Gottesman MM: Mechanisms of cancer drug resistance. Annu Rev Med 53: 615-627, 2002.

24. Adams JM and Cory S: The Bcl-2 protein family: arbiters of cell survival. Science 281: 1322-1326, 1998. 